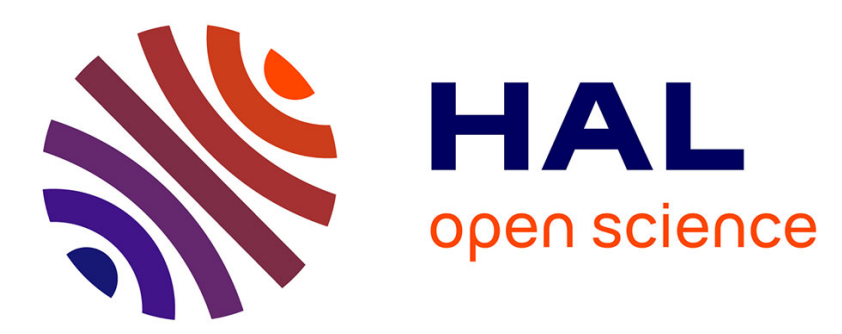

\title{
Effets de la structure du nucléon et des phénomènes de haute énergie sur la diffusion $\mathrm{N}-\mathrm{N}$ à basse énergie
}

Christiane Thévenet

\section{To cite this version:}

Christiane Thévenet. Effets de la structure du nucléon et des phénomènes de haute énergie sur la diffusion N-N à basse énergie. Journal de Physique, 1969, 30 (2-3), pp.148-152. 10.1051/jphys:01969003002-3014800 . jpa-00206772

\section{HAL Id: jpa-00206772 https://hal.science/jpa-00206772}

Submitted on 1 Jan 1969

HAL is a multi-disciplinary open access archive for the deposit and dissemination of scientific research documents, whether they are published or not. The documents may come from teaching and research institutions in France or abroad, or from public or private research centers.
L'archive ouverte pluridisciplinaire HAL, est destinée au dépôt et à la diffusion de documents scientifiques de niveau recherche, publiés ou non, émanant des établissements d'enseignement et de recherche français ou étrangers, des laboratoires publics ou privés. 


\title{
EFFETS DE LA STRUGTURE DU NUGLÉON ET DES PHÉNOMÈNES DE HAUTE ÉNERGIE SUR LA DIFFUSION N-N A BASSE ÉNERGIE
}

\author{
Par Ghristiane THÉVEnet, \\ Institut de Physique Nucléaire, Université de Lyon, France.
}

(Reçu le 22 juillet 1968.)

\begin{abstract}
Résumé. - Dans l'analyse des résultats de la diffusion élastique nucléon-nucléon à basse énergie, on suppose en général que les 5 amplitudes indépendantes sont dominées par l'échange de différents mésons $\pi, \rho, \omega, \sigma, \eta, \varphi$. Cette méthode donne de bons résultats quand la distance entre les nucléons est suffisante $(r \geqslant 0,4 \mathrm{fm})$, tandis que pour $r<0,4 \mathrm{fm}$ on a recours à un cœur dur.

Au lieu d'introduire arbitrairement un cœur dur, nous proposons de modifier les propagateurs associés aux différentes particules échangées de façon à tenir compte des données expérimentales à haute énergie.

Abstract. - The analysis of data on N-N elastic scattering at low energies is usually made by assuming that the five independent amplitudes are dominated by the exchange of the low lying meson resonances as $\pi, \rho, \omega, \sigma, \eta, \varphi$. This method gives good results when the distance between nucleons is not too small $(r \geqslant 0.4 \mathrm{fm})$, while for $r<0.4 \mathrm{fm}$ a "hard core" is used.

In place of an arbitrary hard core description we propose a modification of the propagators associated with the exchange of the different particles, in such a way as to have consistency with the high energy experimental data.
\end{abstract}

Introduction. - Lorsqu'on étudie la diffusion élastique nucléon-nucléon à basse énergie, on suppose en général $[1,2,3]$ que l'interaction est caractérisée par l'échange de pion et de résonances mésiques telles que $\eta, \rho, \omega, \varphi, \sigma$. Les amplitudes d'échange ont été utilisées soit dans les relations de dispersion que l'on résolvait avec la méthode $\mathrm{N} / \mathrm{D}$, soit dans la recherche d'un potentiel [2,3] à insérer dans l'équation de Schrödinger. Le potentiel obtenu n'est pas valable aux faibles distances d'interaction. Aussi suppose-t-on que le nucléon a un « cœur dur ».

Dans ce travail, nous avons essayé de faire la synthèse entre les résultats de la diffusion N-N à haute énergie et le fait qu'à basse énergie l'amplitude (et donc le potentiel) est dominée par l'échange de ces différentes particules. A cet effet, nous modifions les amplitudes d'échange en introduisant un facteur de forme que nous choisissons de façon à tenir compte des données expérimentales à énergie supérieure à $1 \mathrm{GeV}$. Ainsi on évite de recourir à un cœur dur et on essaie de relier la structure interne du nucléon aux phénomènes de haute énergie.

Dans la section II, nous avons discuté du choix du facteur de forme. Puis nous avons montré que la condition de non-violation de l'unitarité pour les am- plitudes d'onde partielle $\left|a_{l}\right|^{2} \leqslant \operatorname{Im} a_{l} \leqslant 1$ pouvait être facilement imposée (sect. III). Enfin, nous avons calculé l'expression du potentiel modifié par l'introduction du facteur de forme.

I. Calcul des amplitudes d'échange et des potentiels. - La méthode utilisée par Wong [3] consiste à chercher des potentiels de façon que l'équation de Schrödinger donne dans l'approximation de Born des amplitudes égales aux amplitudes d'échange des particules telles qu'elles sont utilisées dans les relations de dispersion [1]. Le potentiel ainsi obtenu est, en général, non local et dépend de l'énergie $\mathrm{Il}$ est de la forme :

$$
\begin{aligned}
V=V_{\mathrm{C}}+ & V_{\sigma}\left(\boldsymbol{\sigma}_{\mathbf{1}} \cdot \boldsymbol{\sigma}_{\mathbf{2}}\right)+V_{\mathrm{T}} S_{12} \\
& +V_{\mathrm{LS}} \mathbf{L} . \mathbf{S}+\frac{1}{m^{2}}\left(\boldsymbol{\sigma}_{\mathbf{1}} \cdot \mathbf{p}\right)\left(\boldsymbol{\sigma}_{\mathbf{2}} \cdot \mathbf{p}\right) V_{\mathrm{\sigma p}}
\end{aligned}
$$

où la signification des indices est évidente et où les $V_{i}$ sont des fonctions de $\mathbf{r}, \mathbf{r}^{\prime}$ et $\mathbf{k}$.

En supposant que le potentiel non local s'écrive sous une forme séparable [3] :

$$
V_{i}\left(\mathbf{r}, \mathbf{r}^{\prime}, \mathbf{k}\right)=U_{i}\left(r, k^{2}\right) N\left(\left|\mathbf{r}-\mathbf{r}^{\prime}\right|\right)
$$


on trouve pour l'amplitude de diffusion dans l'approximation de Born :

$f_{\mathbf{B}}(k, \theta)=-\frac{m}{4 \pi} G(k) \int \mathrm{d} \mathbf{r} \mathrm{e}^{i\left(\mathbf{k}^{\prime}-\mathbf{k}\right) \cdot \mathbf{r}} U(\mathbf{r}, s, p) \chi_{\sigma}$

où $\mathbf{k}$ et $\mathbf{k}^{\prime}$ sont les moments linéaires des nucléons dans le système du centre de masse avant et après la collision, $\chi_{\sigma}$ est la fonction de spin et :

$$
G(k)=\int \mathrm{d}\left(\mathbf{r}-\mathbf{r}^{\prime}\right) N\left(\left|\mathbf{r}-\mathbf{r}^{\prime}\right|\right) \mathrm{e}^{i \mathbf{k} \cdot\left(\mathbf{r}-\mathbf{r}^{\prime}\right)} .
$$

Pour chaque état de spin isotopique total, la diffusion nucléon-nucléon est caractérisée par 5 amplitudes indépendantes $M_{i i}$. Nous pouvons choisir, par exemple, l'amplitude de transition entre des états singulets et 4 amplitudes entre états triplets, du spin - 1 au $\operatorname{spin}+1$, de +1 à 0 , de 1 à 1 , de 0 à 0 .

On a :

$$
\begin{aligned}
M_{\mathrm{SS}}= & F_{\mathrm{C}}-3 F_{\sigma}-\frac{k^{2}}{m^{2}} F_{\sigma \mathrm{p}} \\
M_{00}= & F_{\mathrm{C}}+F_{\sigma} \\
& \quad+2 k^{2}(1-\cos \theta)(1-3 \cos \theta) F_{\mathrm{T}}-\frac{k^{2}}{m^{2}} F_{\sigma \mathrm{p}} \\
M_{11}= & F_{\mathrm{C}}+F_{\sigma} \\
& \quad-k^{2}(1-\cos \theta)(1-3 \cos \theta) F_{\mathrm{T}}+\frac{k^{2}}{m^{2}} F_{\sigma \mathrm{p}} \\
M_{1-1}= & -3 k^{2}(1-\cos \theta) F_{\mathrm{T}} \\
M_{10}- & M_{01}=\sqrt{2} k^{2} \sin \theta F_{\mathrm{LS}}
\end{aligned}
$$

avec :

$$
\begin{aligned}
& F_{\mathrm{C}}=-\frac{m^{2}}{E} \int_{0}^{\infty} r^{2} \mathrm{~d} r j_{0}(\Delta r) U_{\mathrm{C}}\left(r, k^{2}\right) \\
& F_{\sigma}=-\frac{m^{2}}{E} \int_{0}^{\infty} r^{2} \mathrm{~d} r j_{0}(\Delta r) U_{\sigma}\left(r, k^{2}\right) \\
& F_{\mathrm{T}}=-\frac{m^{2}}{E} \cdot \frac{1}{\Delta^{2}} \int_{0}^{\infty} r^{2} \mathrm{~d} r j_{2}(\Delta r) U_{\mathrm{T}}\left(r, k^{2}\right) \\
& F_{\mathrm{LS}}=-\frac{m^{2}}{E} \cdot \frac{1}{\Delta} \int_{0}^{\infty} r^{3} \mathrm{~d} r j_{1}(\Delta r) U_{\mathrm{LS}}\left(r, k^{2}\right) \\
& F_{\sigma \mathrm{p}}=-\frac{m^{2}}{E} \int_{0}^{\infty} r^{2} \mathrm{~d} r j_{0}(\Delta r) U_{\sigma \mathrm{p}}\left(r, k^{2}\right) .
\end{aligned}
$$

Dans les équations $(6), \Delta$ est le moment de transfert :

$$
\Delta^{2}=2 k^{2}(1-\cos \theta)=4 k^{2} \sin ^{2} \frac{\theta}{2} \text {. }
$$

Il est facile de calculer les amplitudes d'échange $M_{i i}$ correspondant aux différents mésons en appliquant les règles de Feynmann et d'en déduire les amplitudes $F$ par les formules inverses de (5). Pour le pion par exemple, le lagrangien d'interaction est de la forme :

$$
\mathscr{L}_{\pi}=g_{\pi} \bar{\psi} \gamma_{\mathrm{s}} \tau \psi \boldsymbol{\Phi}_{\pi}
$$

et :

$$
\begin{aligned}
& F_{\mathrm{C}}=F_{\mathrm{LS}}=F_{\sigma \mathrm{p}}=0 \\
& F_{\sigma}=-\frac{g_{\pi}^{2}}{4 E} \cdot \frac{\Delta^{2}}{\Delta^{2}+\mu_{\pi}^{2}} \\
& F_{\mathrm{T}}=\frac{g_{\pi}^{2}}{4 E} \cdot \frac{1}{\Delta^{2}+\mu_{\pi}^{2}}
\end{aligned}
$$

dans le cas où les deux nucléons ont un spin isotopique total $I$ nul. Si $I=1$, on doit multiplier les $F$ par - 1/3. Le même calcul peut être fait pour toutes les particules échangées [3]. En utilisant les formules inverses de (6), on en déduit les potentiels correspondants.

II. Introduction du facteur de forme. - On voit immédiatement les insuffisances de la méthode exposée précédemment puisque, en portant (7) dans (6), on trouve $U_{\sigma}=\infty$. L'origine de ces divergences est évidente, en effet les expressions (7) ont été calculées dans l'approximation de Born. Or, en opérant ainsi, on ne tient compte que de la contribution à l'amplitude due à la partie asymptotique du potentiel (à grande distance), mais on ignore complètement la région d'interaction correspondant aux faibles distances ou, ce qui revient au même, on ignore la structure du nucléon et on le considère comme ponctuel. Pour les deux aspects du problème, il y a désaccord avec l'expérience'. D'une part, la contribution provenant des faibles distances est liée aux effets de haute énergie et nous savons qu'ils sont importants (Wu et Yang [4] ont mis en évidence cette liaison d'un point de vue phénoménologique et ils ont montré que le facteur de forme du nucléon était compatible avec le comportement exponentiel des distributions angulaires trouvées dans les expériences à haute énergie et à grands moments de transfert). D'autre part, il est bien connu [5] que le nucléon ne peut pas être traité comme un objet sans structure.

D'un point de vue purement mathématique, les divergences qui apparaissent dans le calcul du potentiel sont dues au fait que le potentiel est singulier en $r=0$, et que cette singularité ne vérifie pas les conditions du théorème de Fuchs. Il serait malgré tout possible de résoudre les difficultés par des méthodes mathématiques rigoureuses [6]. Mais cette singularité est de nature non physique, elle provient d'une extrapolation jusqu'à de très faibles distances de résultats valables pour $r \rightarrow \infty$. C'est pourquoi de nombreux auteurs, au lieu d'utiliser des techniques mathématiques rigoureuses, recourent à un « cœur dur ». Ceci pourtant est une solution assez peu satisfaisante puisqu'elle revient à considérer le nucléon comme une sphère impénétrable, ce qui constitue une hypothèse un peu simpliste.

Nous choisissons une méthode différente [7]. En effet, les difficultés surgissent du fait que l'on ignore les effets de haute énergie dans la diffusion nucléonnucléon; pour y remédier, il suffit donc de tenir compte 
de ces effets. Au lieu d'utiliser un cœur dur arbitraire, nous modifions les propagateurs associés à l'échange des diverses particules (échange qui est la contribution dominante à basse énergie) de façon à être en accord avec les résultats expérimentaux à haute énergie.

Une modification possible a déjà été étudiée [8] en imposant les conditions suivantes :

1) Chaque potentiel $V_{i}$ doit posséder la symétrie sphérique, doit avoir un faible rayon d'action et doit être moins singulier à l'origine que la barrière centrifuge.

2) L'amplitude à haute énergie pour un angle fixé doit être une fonction exponentiellement décroissante de $s$ (carré de l'énergie dans le système du centre de masse).

3) L'amplitude, pour n'importe quel angle en dehors du pic de diffraction, doit avoir, lorsque $s$ croît, la décroissance la plus rapide permise par les conditions d'analyticité et de borne. En effet, Cerulus et Martin [9] supposent que l'amplitude de diffusion est analytique dans un domaine fixé du plan complexe de la variable $\cos \theta$ contenant la région physique et qu'elle est bornée par $S^{N}$, ils en déduisent que pour des sections efficaces totales lentement variables avec l'énergie l'amplitude à angle fixé ne peut décroître plus vite que :

$$
f(s, \theta) \underset{\substack{s \\ \theta \neq 0, \pi}}{\sim} B \exp \left[b c(\theta) s^{1 / 2} \ln s\right] .
$$

4) Dans la diffusion vers l'avant, l'amplitude doit avoir le comportement typique du pic de diffraction :

$$
f(s, \Delta) \underset{\substack{s \\ \Delta \rightarrow \infty}}{\sim} A \mathrm{e}^{-a \Delta^{2}} .
$$

Luming et Predazzi [8] ont montré que l'amplitude la plus simple qui vérifie ces conditions est de la forme :

$$
f(s, \Delta)=g \frac{K_{\nu}\left[\beta\left(\Delta^{2}+\mu^{2}\right)^{1 / 2}\right]}{\left(\Delta^{2}+\mu^{2}\right)^{v / 2}} .
$$

De plus, Islam et Rosen [10] ont montré que cette expression pouvait être utilisée avec succès pour rendre compte des résultats expérimentaux les plus récents [11] selon lesquels dans la formule de Orear sur les sections efficaces à grand angle de diffusion $\mathrm{d} \sigma / \mathrm{d} \Omega=A \exp \left(-c k_{\mathrm{T}}\right), c$ subit une brusque variation pour : $k_{\mathrm{T}} \simeq 0,8 \mathrm{GeV} / \mathrm{c}$.

Nous pouvons alors supposer que chaque amplitude d'échange des particules soit modifiée par l'introduction d'un facteur de forme :

$$
\varphi_{i}=F_{i}\left(\Delta^{2}\right) \varphi_{\mathrm{B}}^{i}
$$

où $\varphi_{\mathrm{B}}^{i}$ est l'amplitude de Born correspondant à un propagateur libre et où le facteur de forme s'écrit :

$$
F_{i}\left(\Delta^{2}\right)=\beta_{i}\left(\Delta^{2}+\mu_{i}^{2}\right)^{1 / 2} K_{1}\left[\beta_{i}\left(\Delta^{2}+\mu_{i}^{2}\right)^{1 / 2}\right]
$$

et satisfait la condition :

$$
F_{i}\left(-\mu_{i}^{2}\right)=1
$$

afin que l'amplitude se réduise au terme de Born au voisinage du pôle. Dans ce cas, chaque amplitude est de la forme (11), et (9) et (10) sont à peu près satisfaites.

Dans l'équation (13), les $\beta_{i}$ sont des paramètres de régularité et nous supposons que $\operatorname{Re} \beta_{i}>0$. En effet, si $\beta_{i}=0$, le potentiel est singulier comme nous l'avons vu précédemment, au contraire nous verrons que le potentiel est régulier à l'origine lorsque $\operatorname{Re} \beta_{i}>0$.

L'expression du facteur de forme (13) n'est pas la seule possible, mais elle a l'avantage d'être en accord avec un certain nombre de faits expérimentaux à haute énergie, de ne violer a priori aucune condition générale et de ne pas nécessiter l'utilisation d'un « cut-off » dans le calcul du potentiel. D'autre part, elle conduit pour le potentiel à des calculs relativement simples.

III. Considérations sur les ondes partielles. L'introduction du facteur de forme $F\left(\Delta^{2}\right)$ apporte une amélioration à la simple approximation de Born en ce qui concerne la non-violation des conditions d'unitarité. En effet, montrons qu'en imposant certaines conditions aux paramètres $\beta_{i}$, les amplitudes d'onde partielle vérifient $\left|a_{l}\right| \leqslant 1$.

Considérons par exemple le cas du pion, supposons $\beta_{\pi}$ réel :

$F_{\sigma}=-\frac{g_{\pi}^{2}}{4 E} \cdot \frac{\Delta^{2}}{\left(\Delta^{2}+\mu_{\pi}^{2}\right)^{1 / 2}} \cdot K_{1}\left[\beta_{\pi}\left(\Delta^{2}+\mu_{\pi}^{2}\right)^{1 / 2}\right]$.

Par définition, on a :

$$
a_{l}=\frac{i k}{2} \int_{-1}^{1} \mathrm{~d}(\cos \theta) F_{\sigma} P_{l}(\cos \theta) .
$$

En tenant compte des inégalités :

$$
\begin{gathered}
\left|P_{l}(\cos \theta)\right| \leqslant 1 \\
\frac{\Delta^{2}}{\Delta^{2}+\mu_{\pi}^{2}} \leqslant \frac{4 k^{2}}{4 k^{2}+\mu_{\pi}^{2}}
\end{gathered}
$$

et des formules de dérivation des fonctions de Bessel modifiées, on trouve :

$\left|a_{l}\right| \leqslant \frac{g_{\pi}^{2} k}{2 E}\left[\frac{\mu_{\pi}^{2}}{4 k^{2}+\mu_{\pi}^{2}} K_{2}\left(\beta_{\pi} \mu_{\pi}\right)-K_{2}\left[\beta_{\pi}\left(4 k^{2}+\mu_{\pi}^{2}\right)^{1 / 2}\right]\right]$.

Il est alors facile de vérifier que si $\beta_{\pi} \mu_{\pi} \geqslant 0,25$, $\left|a_{l}\right| \leqslant 1$ si l'énergie cinétique du nucléon incident est inférieure à $400 \mathrm{MeV}$ et $a_{l} \underset{k \rightarrow \infty}{\rightarrow} 0$.

Si $\beta_{\pi}$ est complexe, le calcul précédent est encore valable à condition de remplacer dans l'argument de la fonction $K_{1}, \beta_{\pi}$ par $\operatorname{Re} \beta_{\pi}$ puisque :

$$
K_{v}(z)=\int_{0}^{\infty} \mathrm{e}^{-z \operatorname{ch} t} \operatorname{ch}(v t) \mathrm{d} t \quad(\operatorname{Re} z>0)
$$

et on obtient :

$$
\begin{aligned}
\left|a_{l}\right| \leqslant \frac{g_{\pi}^{2} k}{2 E} \cdot \frac{\left|\beta_{\pi}\right|}{\operatorname{Re} \beta_{\pi}}\left[\frac{\mu_{\pi}^{2}}{4 k^{2}+} \mu_{\pi}^{2}\right. & K_{2}\left(\operatorname{Re} \beta_{\pi} \cdot \mu_{\pi}\right) \\
& \left.\quad-K_{2}\left[\operatorname{Re} \beta_{\pi}\left(4 k^{2}+\mu_{\pi}^{2}\right)^{1 / 2}\right]\right] .
\end{aligned}
$$


Et en choisissant judicieusement les parties réelle et imaginaire de $\beta_{\pi}$, on pourra satisfaire la double condition :

$$
\left|a_{l}\right|^{2} \leqslant \operatorname{Im} a_{l} \leqslant 1
$$

On peut montrer de la même façon que les conditions de non-violation de l'unitarité sont remplies pour les autres amplitudes d'échange en choisissant convenablement les parties réelles et imaginaires de $\beta_{i}$.

Donnons maintenant les expressions des amplitudes d'onde partielle.

Supposons que l'amplitude d'échange soit de la forme :

$$
F=A \beta\left(\Delta^{2}+\mu^{2}\right)^{1 / 2} K_{1}\left[\beta\left(\Delta^{2}+\mu^{2}\right)^{1 / 2}\right] .
$$

Nous pouvons écrire sa décomposition en $C_{n}^{1}(\cos \theta)$ [12] (HTF, II, p. 43) :

$F=\frac{2 A}{k^{2}} \sum_{n=0}^{\infty}(n+1) C_{n}^{1}(\cos \theta) I_{n+1}\left(\beta r_{0}\right) K_{n+1}(\beta r)$

avec :

$$
\begin{aligned}
& r=\frac{\sqrt{4 k^{2}+\mu^{2}}+\mu}{2} \\
& r_{0}=\frac{\sqrt{4 k^{2}+\mu^{2}}-\mu}{2}
\end{aligned}
$$

Comme $C_{n}^{1}(\cos \theta)=\frac{\sin (n+1) \theta}{\sin \theta}$, il est facile de décomposer $F$ en polynômes de Legendre et on trouve :

$a_{l}=\frac{A}{k} \sum_{m=0}^{\infty}(l+2 m+1)$

$\frac{F(m+1 / 2) F(m+l+1)}{F(m+1) F(m+l+3 / 2)} I_{l+2 m+1}\left(\beta r_{0}\right) K_{l+2 m+1}(\beta r)$.

Lorsque $k^{2} \rightarrow 0$, la condition de seuil est vérifiée puisque l'équation (21) donne :

$a_{l} \underset{k^{2} \rightarrow 0}{\sim} A \sqrt{\pi} K_{l+1}(\beta \mu) \frac{1}{F(l+3 / 2)}\left(\frac{\beta}{2 \mu}\right)^{l+1} k^{2 l+1}$.

Ainsi nous sommes en mesure de calculer les amplitudes d'onde partielle correspondant aux amplitudes d'échange de chaque particule (pour les expressions de l'amplitude différentes de (19), voir référence [13]). Voyons maintenant quelles sont les expressions des potentiels correspondants.

IV. Calcul des potentiels. - Les amplitudes d'échange des particules calculées dans l'approximation de Born telles que (7) sont multipliées par le facteur de forme (13), les équations (6) inversées conduisent alors à des intégrales du type :

$I_{p, \nu}=\int_{0}^{\infty} \frac{K_{1}\left[\beta\left(\Delta^{2}+\mu^{2}\right)^{1 / 2}\right]}{\left(\Delta^{2}+\mu^{2}\right)^{1 / 2}} \Delta^{2 p+\nu+1} J_{\nu}(\Delta r) \mathrm{d} \Delta$.
Les formules de dérivation des fonctions de Bessel $J$ et $K$ donnent :

$\Delta^{2 p+\nu+1} J_{\nu}(\Delta r)=r^{-p-\nu} \frac{\partial^{p}}{\partial r^{p}}\left[\Delta^{p+\nu+1} r^{p+\nu} J_{p+\nu}(\Delta r)\right]$

$\frac{K_{1}\left[\beta\left(\Delta^{2}+\mu^{2}\right)^{1 / 2}\right]}{\left(\Delta^{2}+\mu^{2}\right)^{1 / 2}}=(-1)^{p+\nu-1 / 2} \beta^{-p-\nu-1 / 2} \frac{\partial^{p+\nu-1 / 2}}{\partial \beta^{p+\nu-1 / 2}}$
$\left[\beta^{p+\nu+1 / 2}\left(\Delta^{2}+\mu^{2}\right)^{(p+\nu) / 2+1 / 4} K_{p+\nu+1 / 2}\left[\beta\left(\Delta^{2}+\mu^{2}\right)^{1 / 2}\right]\right]$.

Et nous avons :

$I_{p, \nu}=(-1)^{p+\nu-1 / 2} \beta^{-p-\nu-1 / 2} r^{-p-\nu} \frac{\partial^{2 p+\nu-1 / 2}}{\partial r^{p} \partial \beta^{p+\nu-1 / 2}} r^{p+\nu-1 / 2}$ $\times \int_{0}^{\infty} \beta^{p+\nu+1 / 2}\left(\Delta^{2}+\mu^{2}\right)^{(-p+\nu) / 2} K_{p+\nu+1 / 2}$

$$
\left[\beta\left(\Delta^{2}+\mu^{2}\right)^{1 / 2}\right] J_{\nu+p}(\Delta r) \mathrm{d} \Delta .
$$

En intégrant cette expression :

$$
\begin{gathered}
I_{p, \nu}=(-1)^{p+\nu-1 / 2} \sqrt{\frac{\pi}{2} \beta^{-p-\nu-1 / 2} r^{-p-\nu} \frac{\partial^{2 p+\nu-1 / 2}}{\partial r^{p} \partial \beta^{p+\nu-1 / 2}}} \\
{\left[r^{2(p+\nu)} \frac{\mathrm{e}^{-\mu\left(\beta^{2}+r^{2}\right)^{1 / 2}}}{\left(\beta^{2}+r^{2}\right)^{1 / 2}}\right]}
\end{gathered}
$$

et en utilisant la formule (27) pour chaque amplitude d'échange on obtient les différents potentiels [13]. Même si les expressions ainsi obtenues sont assez compliquées dans leur forme, elles peuvent en réalité être utilisées sans grande difficulté dans un calcul numérique puisqu'il s'agit simplement d'une formule de récurrence

Conclusion. - Les résultats peuvent être résumés ainsi :

1) Tous les potentiels sont réguliers en $r=0$, il n'est donc pas nécessaire de recourir au « cœur dur ». De plus, ces potentiels appartiennent à une des deux classes générales de potentiel dont Cottingham et Dombey [14] ont montré que ce sont les seules qui reproduisent le comportement expérimental des amplitudes à haute énergie.

2) On peut calculer l'amplitude d'ondes partielles et on peut voir en particulier que le comportement au seuil $k=0$ est satisfait

3) Les conditions de non-violation de l'unitarité $\left|a_{l}\right|^{2} \leqslant \operatorname{Im} a_{l} \leqslant 1$ peuvent être facilement vérifiées et il en résulte certaines conditions sur les coefficients de régularité $\beta_{i}$.

4) Les potentiels obtenus sont suffisamment simples pour être effectivement utilisés dans une résolution numérique de l'équation de Schrödinger de façon à reproduire les données de basse énergie.

Nous avons introduit un paramètre de régularité $\beta$ pour chaque particule échangée. Ces paramètres ne sont pas arbitraires, ils ne peuvent varier que dans 
des limites bien déterminées de façon à respecter les conditions suivantes :

- L'amplitude à haute énergie vers l'avant qui est automatiquement de la forme (10) est à prédominance imaginaire; son module et sa pente sont connus expérimentalement.

- Pour de grands angles de diffusion, l'amplitude admet des discontinuités qu'il est facile d'interpréter. En effet, chaque échange de particules domine dans une région donnée du domaine de haute énergie, comme les paramètres de régularité sont différents suivant les particules échangées, la pente de la distribution angulaire varie.

- La section efficace totale doit tendre vers une constante lorsque l'énergie croît.

Une fois que l'on a imposé ces conditions aux paramètres $\beta$, on les ajuste grâce aux données expérimen- tales de basse énergie après résolution numérique de l'équation de Schrödinger.

Bien que le présent modèle soit encore très phénoménologique, son grand avantage réside dans le fait qu'on a essayé de relier les effets de haute et basse énergies. Nous espérons conduire l'analyse jusqu'à une comparaison numérique avec l'expérience de façon à tester la validité de la méthode.

Il en résulterait de plus une meilleure connaissance de la structure du nucléon et de son incidence sur les phénomènes de haute énergie.

Remerciements. - Je remercie le Professeur Fubini de m'avoir accueillie à l'Institut de Physique Théorique de Turin et le Professeur Predazzi qui m'a proposé ce sujet et m'a aidée de ses précieux conseils tout au long de ce travail.

\section{BIBLIOGRAPHIE}

[1] Scotrit (A.) et Wong (D. Y.), Phys. Rev., 1965 $138 \mathrm{~B}, 145$

BALL (J. S.), ScotTi (A.) et Wong (D. Y.), Phys. Rev., 1966, $142 \mathrm{~B}, 1000$

[2] Bryan (R. A.), Dismukfis (C. R.) et Ramsay (W.), Nucl. Phys., 1963, 45, 353.

HOSHIZAKI (N.), OTSUKI (S.), WATARI (W.) et Yonezawa (M.), Prog. Theor. Phys., 1961, 29, 680.

Bryan (R. A.) et Scot'T (B. L.), Phys. Rev., 1964, $135 \mathrm{~B}, 434$

Bryan (R. A.) et ARNDT (R. A.), Phys. Rev., 1966 $150 \mathrm{~A}, 1299$

[3] Wong (D. Y.), Nucl. Phys., 1964, 55, 212.

[4] WU (R. R.) et YANG (C. N.), Phys. Rev., 1965, $137 \mathrm{~B}, 708$.

[5] Hofstadter(R.), Suppl. Nuovo Cimento, 1965, 3, 568.

[6] Voir par exemple : CORNILLE (H.) et PREDAzzI (E.), Nuovo Cimento, 1965, 35, 879; pour une bibliographie complète, voir : CALOGERo (F.), Phys. Rev., 1966, 146, 1196.
[7] Thévenet (C.) et Predazzi (E.), C. R. Acad. Sc. (à paraître)

[8] Luming (M.) et PREdazzI (E.), Rapport de l'Université de Chicago, EFINS-66.25 (non publié).

[9] Cerrulus (F.) et Martin (A.), Phys. Lett., 1964, 8, 80.

[10] Islam (M. M.) et Rosen (J.), Phys. Rev. Lett. 1967, 19, 178.

[11] Allaby (J. V.) et Belletini (G.) et al., Phys. Lett., 1966, 23, 389 .

AkerloF (C. V.) et Hieber (R. M.) et al., Phys. Lett., 1966, 17, 1105.

Krisch (A. D.), Phys. Rev. Lett., 1967, 19, 1149.

[12] BATEMAN, Higher Transcendental Functions (HTF), Tables of Integral Transformations (TIT).

[13] Thévenet (C.), Thèse Doct. Spécialité $3^{\mathrm{e}}$ cycle, Lyon, 1968

[14] Cotitingham (W. N.) et Dombey (N.), Nuovo Cimento, 1966, 48 A, 397. 